\title{
CONGENITAL LUNG DISPLACEMENT IN AN ENGLISH BULLDOG PUPPY
}

\author{
Alessia Gloria, Giuseppe MARrUCHELla, Lucia Di FrANCESCO and Alberto CONTRI* \\ University of Teramo, Faculty of Veterinary Medicine, Veterinary Teaching Hospital, \\ Loc. Piano d'Accio, 64100 Teramo, Italy
}

(Received 28 July 2019; accepted 19 October 2019)

\begin{abstract}
The English bulldog is notoriously predisposed to a number of disease conditions, including congenital defects, likely due to extreme genetic selection which has reduced genetic variability within this breed. The present report describes, for the first time, the main clinical and pathological features of congenital lung displacement in an English bulldog puppy. The defect involved the entire left lung, which was normally developed but rotated and shifted to the right hemithorax. The pathogenesis of congenital anomalies is largely unknown. Characterisation of the genetic bases of these malformations is crucial for a reform of the breeding selection which is also aimed at correcting phenotypic abnormalities with a strong impact on individual health.
\end{abstract}

Key words: Breeding, congenital anomalies, dog, heterotopic lung

Several problems have recently been shown to be related to genetic health in purebred dogs. Among these, the English bulldog shows a very low genetic diversity, which raises many health issues and results in a shorter lifespan when compared with other canine breeds (O'Neill et al., 2013; Pedersen et al., 2016). In particular, the English bulldog is known to be predisposed to a number of disease conditions, such as brachycephalic syndrome, chondrodysplasia, prognathism, dental disorders, cataract, heart defects, immunologic disorders, and cancer (Pedersen et al., 2016). This breed ranks second in fatal congenital diseases after the Newfoundland, with a high incidence of flat chest with splayed legs, anasarca and cleft palate (Fleming et al., 2011). In addition, changes in skeletal conformation are also frequently observed in English bulldogs and can complicate copulation/parturition, thus making artificial insemination and Caesarean section often necessary (Fleming et al., 2011; O’Neill et al., 2013).

The aim of the present manuscript is to describe, for the first time, the main clinical and pathological features of a case of congenital lung displacement in an English bulldog puppy.

\footnotetext{
*Corresponding author; E-mail: acontri@unite.it; Phone/Fax: 0039 (0861) 266-995
} 


\section{Case description}

A 30-month-old female English bulldog was presented for routine reproductive monitoring at the Veterinary Teaching Hospital of the University of Teramo. The animal was managed in agreement with the rules of the Italian legislation concerning animal care (Italian Legislative Decree LD n. 116, 27/01/ 1992). The owner reported a history of infertility. Haematological and biochemical analysis as well as ultrasonographic examination were carried out to rule out systemic and reproductive diseases, and no alterations were found.

The bitch was monitored through vaginal cytology and for progesteronaemia (by radioimmunoassay) to define the oestrous cycle and ovulation, assumed as the day with blood progesterone of $6-8 \mathrm{ng} / \mathrm{mL}$. Two artificial inseminations were performed at days 1 and 3 after ovulation, with fresh semen collected from an English bulldog male of proven fertility. Semen evaluations were performed by a computer-assisted sperm analyser (CASA) IVOS 12.3 (HamiltonThorne Bioscience, Beverly, MA, USA) for motility and an Olympus BX51 epifluorescence microscope (Olympus Italy, Milan, Italy) for membrane integrity, and no alterations were recorded.

Ultrasonography at day 24 after ovulation confirmed the pregnancy. Thereafter, ultrasonographic evaluations were performed at 15-day intervals to assess fetal viability.

The bitch was submitted for elective Caesarean section at 62 days after ovulation, with a progesterone value of $1.87 \mathrm{ng} / \mathrm{mL}$. Five puppies were recovered, out of which one was mummified. Another puppy was affected by anasarca and died 4 hours post-partum, while the remaining three puppies were apparently healthy. During the following days, a female puppy became weak and showed a reduction in suckling time, worsening respiratory distress, and progressive weight loss. On clinical examination, the conjunctival mucosa was hyperaemic, the body temperature was $38.4{ }^{\circ} \mathrm{C}$, the heart rate was 200 beats/min, and the breathing frequency was $60 \mathrm{breaths} / \mathrm{min}$. Antimicrobial therapy was implemented with ceftriaxone administered subcutaneously ( $25 \mathrm{mg} / \mathrm{kg}$ BID), and the puppy was clinically monitored every 4 days. Despite this treatment, the clinical condition deteriorated and the puppy died at 25 days of age and was then submitted for necropsy.

At the external examination, no relevant abnormality was revealed. Likewise, no gross lesions seemed to affect the peritoneal cavity and the abdominal organs. A remarkable alteration of the topography of thoracic organs was seen after opening the chest cavity. The pericardium and the heart almost completely occupied the left hemithorax, no lung parenchyma being detected at that level, while a lung was observed inside the right hemithorax (Fig. 1). Actually, at a closer view, a small portion of another lung was seen, protruding caudally. After the removal of the thoracic organs, it became clear that both lungs were present (Fig. 2). The right lung corresponded to the most visible one after opening the 
chest, and it appeared larger when compared with the left one. The right lung showed a non-homogeneous appearance, with emphysematous areas of lighter colour and cottony consistency intermingled with slightly depressed and hyperaemic areas. The left lung corresponded to the small portion of parenchyma protruding caudally to the right one, as shown in Fig. 1. As a matter of fact, it was rotated by 180 degrees and shifted to the right hemithorax and appeared smaller and hyperaemic, most likely due to the compression between the right lung and the chest wall. The pulmonary lobes and the ramification of the bronchial tree were normal. The heart appeared considerably enlarged and round-shaped due to the hypertrophy of the right ventricle. The examination of the cardiac chambers and valves did not demonstrate any congenital defects. No further gross lesions were detected.

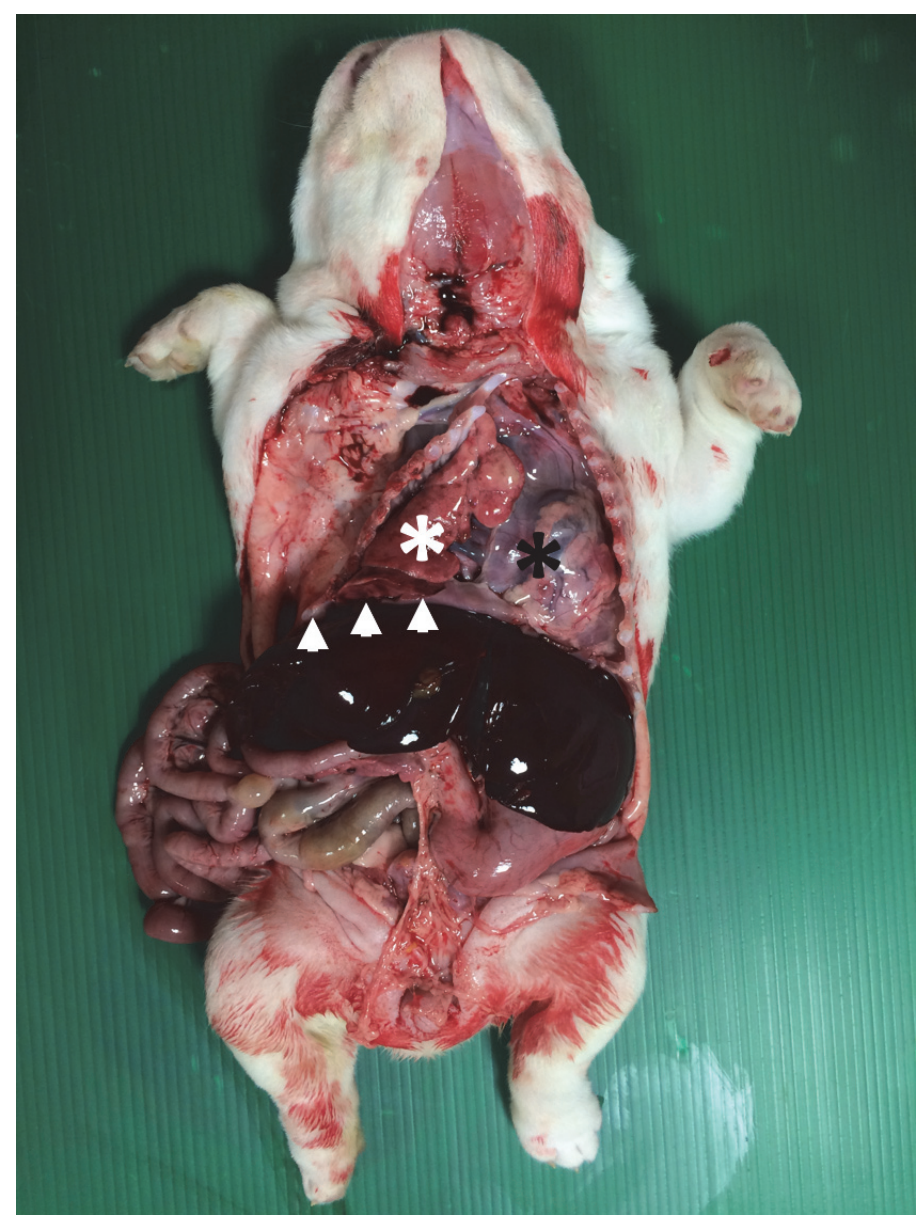

Fig. 1. English bulldog puppy. Necropsy. In the left hemithorax, the pericardial sac is only seen (black asterisk). On the other side, two lungs are present (white asterisk and arrowheads) 


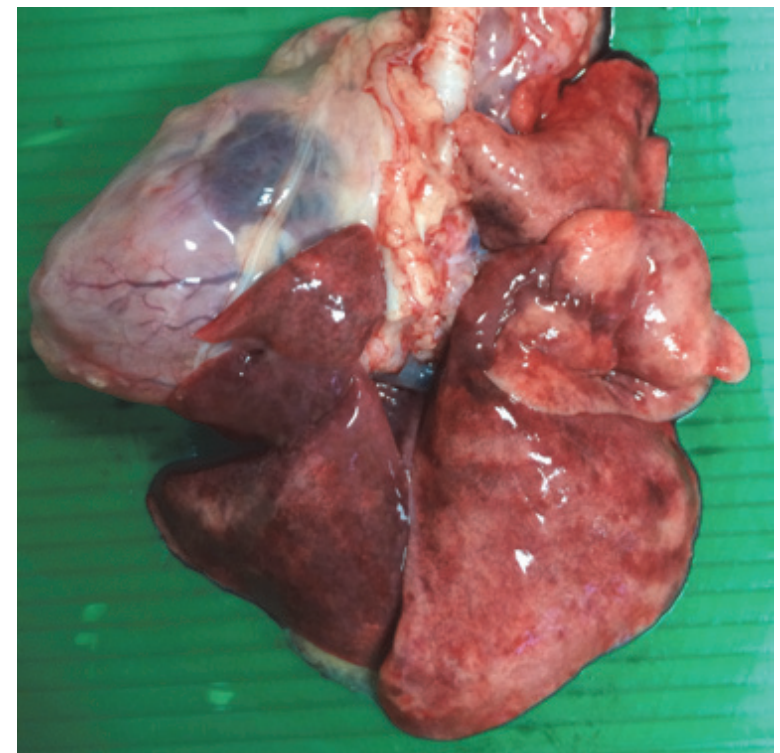

Fig. 2. English bulldog puppy. Lungs. The lobation of both lungs is normal. The right lung appears larger and shows a non-homogeneous appearance, with intermingled emphysematous and depressed areas. The left lung seems smaller and is diffusely oedematous and hyperaemic

All organs were sampled, fixed in $10 \%$ neutral buffered formalin, embedded in paraffin and routinely processed for histological investigations (haematoxylin and eosin stain). Microscopically, the left lung appeared severely hyperaemic with a marked reduction of the alveolar spaces, which were often filled by a dense infiltration by macrophages, while the right lung was mainly emphysematous. The bronchial cartilage was normally developed. No relevant microscopic lesions affected the other organs. Based on these findings, the diagnosis of congenital displacement of the left lung was made.

\section{Discussion}

The incidence of congenital defects reported in the English bulldog is high, ranging between $6.95 \%$ and $8.2 \%$ (Wydooghe et al., 2013; Batista et al., 2014). Such data mostly refer to malformations clearly evident during external examination of the body - e.g. anasarca (Wydooghe et al., 2013; Batista et al., 2014), cleft palate (Wydooghe et al., 2013; Batista et al., 2014) and severe fontanelle (Batista et al., 2014) -, thus the real prevalence of congenital disorders is probably underestimated. This high frequency of congenital defects is possibly due to the limited number of founders of this breed, as well as to extreme genetic selection (Pedersen et al., 2016). 
In this case report, the macroscopic and microscopic evaluation of the lung supported the diagnosis of congenital lung displacement in the puppy. The morphological abnormality to the right ventricle was likely the result of an alteration of pulmonary haemodynamics, secondary to the modified topography of the thoracic organs. To the best of the authors' knowledge, this is the first report of congenital lung displacement in mammals and particularly in the English bulldog. This malformation involved an entire, otherwise normal and well-developed lung, and lengthens the list of pulmonary malformations so far described.

Inherited and congenital anomalies of the lower respiratory tract are generally rare in all animal species and mainly result from the abnormal development of the lung bud and/or of the pulmonary circulation. Major malformations are incompatible with life and can coexist with other anomalies (Caswell and Williams, 2007). In dogs, primary ciliary dyskinesia ('Kartagener syndrome') is the condition most frequently reported; other abnormalities include an anomalous tracheobronchial tree, bronchial cartilage hypoplasia with lobar congenital emphysema, broncho-oesophageal fistulas, pulmonary vascular anomalies, and accessory lungs (Caswell et al., 2007). As far as pulmonary displacement is concerned, the lung lobe torsion around the bronchus and vascular supply have also been described as a rare, acquired, life-threatening condition in small animals. It most frequently affects large deep-chested dogs (e.g. Afghan Hounds), sometimes secondary to an underlying pathology (Caswell et al., 2007; Gicking and Aumann, 2011).

This report underlines the importance of diagnostic investigations, including necropsy, to better estimate the real prevalence of congenital anomalies and to add knowledge about their peculiar characteristics. This is especially true for malformations affecting the internal organs which are not seen at the external inspection of the body.

Among the causes of malformations, toxic and teratogenic substances or drugs administered during gestation should also be considered. Gestational administration of prednisone and doxycycline was associated with multiple cases of midline and cardiac defects in Golden Retriever puppies (Kaplan et al., 2018). In the present case, no administration of drugs either before or during the gestation of the bitch was performed. Also, the bitch lives in the house together with the owner and his family, under continuous surveillance.

In conclusion, the present case report describes, for the first time, a rare congenital pulmonary abnormality of the English bulldog. This finding underlines the importance of necropsy of the puppy, especially in at-risk breeds, and the need to quantify the real prevalence of these diseases of genetic basis. Indeed, this represents the first step for a genetic-based breeding reform aimed at correcting phenotypic abnormalities that strongly impact on individual health (O'Neill et al., 2013; Pedersen et al., 2016). 


\section{References}

Batista, M., Moreno, C., Vilar, J., Golding, M., Brito, C., Santana, M. and Alamo, D. (2014): Neonatal viability evaluation by Apgar score in puppies delivered by cesarean section in two brachycephalic breeds (English and French bulldog). Anim. Reprod. Sci. 146, 218-226.

Caswell, J. L. and Williams, K. J. (2007): Respiratory system. In: Maxie, G. (ed.) Jubb, Kennedy \& Palmer's Pathology of Domestic Animals: Volume 1, 5th Edition. Saunders Ltd., Philadelphia, PA, USA.

Fleming, J. M., Creevy, K. E. and Promislow, D. E. (2011): Mortality in North American dogs from 1984 to 2004: an investigation into age-, size-, and breed-related causes of death. J. Vet. Intern. Med. 25, 187-189.

Gicking, J. and Aumann, M. (2011): Lung lobe torsion. Compend. Contin. Educ. Vet. 33, E4.

Kaplan, J. L., Gunther-Harrington, C. T., Sutton, J. S. and Stern, J. A. (2018): Multiple midline defects identified in a litter of golden retrievers following gestational administration of prednisone and doxycycline: a case series. BMC Vet. Res. 14, 86.

O’Neill, D. G., Church, D. B., McGreevy, P. D., Thomson, P. C. and Brodbelt, D. C. (2013): Longevity and mortality of owned dogs in England. The Vet. J. 198, 638-643.

Pedersen, N. C., Pooch, A. S. and Liu, H. (2016): A genetic assessment of the English bulldog. Canine Genet. Epidemiol. 3, 6.

Wydooghe, E., Bergmans, E., Rijsselaere, T. and Van Soom, A. (2013): International breeder inquiry into the reproduction of the English bulldog. Vlaams Diergeneeskd. Tijdschr. 82, 38-43. 